\title{
EXTENDING EDGAR'S ORDERING TO LOCALLY CONVEX SPACES
}

\author{
by NEILL ROBERTSON
}

(Received 20 November, 1990)

By the term "locally convex space", we mean a locally convex Hausdorff topological vector space (see [17]). We shall denote the algebraic dual of a locally convex space $E$ by $E^{*}$, and its topological dual by $E^{\prime}$. It is convenient to think of the elements of $E$ as being linear functionals on $E^{\prime}$, so that $E$ can be identified with a subspace of $E^{\prime *}$. The adjoint of a continuous linear map $T: E \rightarrow F$ will be denoted by $T^{\prime}: F^{\prime} \rightarrow E^{\prime}$. If $\langle E, F\rangle$ is a dual pair of vector spaces, then we shall denote the corresponding weak, strong and Mackey topologies on $E$ by $\sigma(E, F), \beta(E, F)$ and $\mu(E, F)$ respectively.

1. The relation $<$. In [8], G. A. Edgar introduced an intriguing binary relation on the class of Banach spaces; for any pair of Banach spaces $E, F$, he defined $E<F$ to mean that for each $\phi \in E^{\prime \prime} \backslash E$, there is a continuous linear map $T: E \rightarrow F$ with $T^{\prime \prime}(\phi) \in F^{\prime \prime} \backslash F$. This relation is commonly referred to as Edgar's ordering (see [5, p. 115]). Edgar showed how a wide range of Banach space properties could be expressed in terms of his ordering. Although he noted the possibility of extending $<$ to the class of locally convex spaces, he did not do so.

One can apply the above definition to locally convex spaces, provided that one first chooses a suitable topology on $E^{\prime}$. A natural choice is the topology $\beta\left(E^{\prime}, E\right)$, so that $E^{\prime \prime}$ is the usual space of $\beta\left(E^{\prime}, E\right)$-continuous linear functionals on $E^{\prime}$. However, a problem arises, in that the completion of a locally convex space $E$ need not be a subspace of $E^{\prime \prime}$. To avoid this difficulty, we shall modify the definition of $<$ slightly, and replace the biduals $E^{\prime \prime}$ and $F^{\prime \prime}$ by the algebraic duals of $E^{\prime}$ and $F^{\prime}$ respectively. We thus have the following definition.

Definition. For each pair of locally convex spaces $E, F$, we define $E<F$ to mean that each $\phi \in E^{\prime *}$ satisfies one of the following conditions: either $\phi \in E$, or there exists a continuous linear map $T: E \rightarrow F$ such that $\phi \circ T^{\prime}$ fails to be $\sigma\left(F^{\prime}, F\right)$-continuous on $F^{\prime}$.

Edgar's ordering has strong connections with the notion of a Tauberian operator [19]. Recall that if $E$ and $F$ are Banach spaces, then a continuous linear map $T: E \rightarrow F$ is said to be a Tauberian operator if $\phi \notin E \Rightarrow T^{\prime \prime}(\phi) \notin F$ for all $\phi \in E^{\prime \prime}$. The existence of a Tauberian operator $T: E \rightarrow F$ ensures that $E<F$. It can be seen that many properties of Tauberian operators carry over to the relation $<$. For example, N. Kalton and A. Wilansky have shown [19,3.2] that if $E$ and $F$ are Banach spaces, then a continuous linear map $T: E \rightarrow F$ is a Tauberian operator if and only if the following statements are equivalent for each subset $A$ of $E$ :

(1) $A$ is relatively $\sigma\left(E, E^{\prime}\right)$-compact, and

(2) $A$ is bounded and $T(A)$ is a relatively $\sigma\left(F, F^{\prime}\right)$-compact subset of $F$.

Similar results appear in more recent work by R. D. Neidinger (e.g., [21, Lemma 5]). In the case of $<$, we have the following result. 
Proposition 1.1. Let $E$ and $F$ be locally convex spaces with $E<F$. For each subset $A$ of $E$, the following statements are equivalent:

(1) $A$ is relatively $\sigma\left(E, E^{\prime}\right)$-compact, and

(2) $A$ is bounded and $T(A)$ is a relatively $\sigma\left(F, F^{\prime}\right)$-compact subset of $F$ for each continuous linear map $T: E \rightarrow F$.

Proof. The implication (1) $\Rightarrow(2)$ is trivial. Suppose that $A$ satisfies condition (2). Let $B$ be the $\sigma\left(E^{\prime \prime}, E^{\prime}\right)$-closure of $A$ in $E^{\prime \prime}$. Because $A$ is bounded, $B$ is $\sigma\left(E^{\prime \prime}, E^{\prime}\right)$-compact. We wish to show that $B \subseteq E$. Suppose that there exists a $\phi \in B$ that does not belong to $E$. Since $E<F$, there must be a continuous linear map $T: E \rightarrow F$ with $T^{\prime \prime}(\phi) \notin F$. But because $T(A)$ is relatively $\sigma\left(F, F^{\prime}\right)$-compact, $T^{\prime \prime}(\phi)$ must lie in the $\sigma\left(F, F^{\prime}\right)$-closure of $T(A)$ in $F$, a contradiction.

Recall that a locally convex space $E$ is semireflexive if and only if the bounded absolutely convex subsets of $E$ are relatively $\sigma\left(E, E^{\prime}\right)$-compact.

Corollary 1.2. Let $E$ and $F$ be locally convex spaces with $E<F$. If $F$ is semireflexive, then so is $E$.

The above Corollary generalises [8, Proposition 1]. The following simple observation is also closely related to Proposition 1.1.

Proposition 1.3. Let $E$ and $F$ be locally convex spaces with $E<F$. If $F$ is complete, then so is $E$.

Proof. Let $\phi$ be a linear functional that manages to be $\sigma\left(E^{\prime}, E\right)$-continuous on the equicontinuous absolutely convex subset of $E^{\prime}$. We wish to show that $\phi \in E$. For each continuous linear map $T: E \rightarrow F$, the linear functional $\phi \circ T^{\prime}$ is $\sigma\left(F^{\prime}, F\right)$-continuous on the equicontinuous absolutely convex subsets of $F^{\prime}$. According to Grothendieck's Completeness Theorem $[\mathbf{1 7}, 9.2 .2]$, such a functional belongs to $F$. Since $E<F$, this means that $\phi \in E$, as required.

This Proposition becomes particularly powerful when linked to the fact that the relation $<$ is "functorial". By this we mean that $E<F$ if and only if the same relationship holds when the topologies on $E$ and $F$ are replaced by any other pair of locally convex topologies inducing the same set of continuous linear maps. In particular, we have that $E<F$ if and only if the same relationship holds when the given topologies on $E$ and $F$ are replaced simultaneously by the weak topologies $\sigma\left(E, E^{\prime}\right)$ and $\sigma\left(F, F^{\prime}\right)$, or by the Mackey topologies $\mu\left(E, E^{\prime}\right)$ and $\mu\left(F, F^{\prime}\right)$.

2. The condition of Mazur. A Banach space $E$ is said to satisfy the condition of Mazur if the $\sigma\left(E^{\prime}, E\right)$-sequentially continuous linear functionals on $E^{\prime}$ are $\sigma\left(E^{\prime}, E\right)$ continuous. One of the many interesting results in Edgar's paper [8] is that a Banach space $E$ satisfies the condition of Mazur if and only if $E<c_{0}$.

In order to extend this result, we shall show that Banach spaces satisfying the condition of Mazur are related to a very large and well-known class of locally convex spaces. A locally convex space $E$ is said to be separable by seminorm if for each neighbourhood $U$ of 0 in $E$ there is a countable subset $C$ of $E$ with $E=C+U$. This idea appears under various names in the literature; Pfister [22] says that such a space is "of 
countable type", while Drewnowski [6] dubs $E$ "transseparable". The terminology "separable by seminorm" can be traced back to [13].

Any separable locally convex space is separable by seminorm. The converse does not hold in general, but is true for metrisable spaces. Separability by seminorm enjoys good permancence properties: it is preserved under the taking of completions, subspaces, products, continuous linear images and projective limits.

The following characterisation is fundamental; it can be found in [22].

Proposition 2.1. A locally convex space $E$ is separable by seminorm if and only if the equicontinuous subsets of $E^{\prime}$ are $\sigma\left(E^{\prime}, E\right)$-metrisable.

It follows that if a locally convex space $E$ is equipped with its weak topology $\sigma\left(E, E^{\prime}\right)$, then it is separable by seminorm. This means that unlike ordinary separability, separability by seminorm is not a duality invariant: a locally convex space $E$ that fails to be separable by seminorm when equipped with its given topology becomes separable by seminorm when equipped with $\sigma\left(E, E^{\prime}\right)$.

The class of locally convex spaces which are separable by seminorm is very large. From Proposition 2.1 we can see that it includes all Schwartz spaces. More generally, a locially convex space $E$ is said to be semi-weak [2] if every equicontinuous subset of $E^{\prime}$ is contained in the $\sigma\left(E^{\prime}, E\right)$-closed absolutely convex hull of an equicontinuous $\sigma\left(E^{\prime}, E\right)$ null sequence. It can be seen that the class of semi-weak locally convex spaces includes the Schwartz spaces; the following Lemma shows that every semi-weak space is separable by seminorm.

Lemma 2.2. Let $E$ be a locally convex space. The $\sigma\left(E^{\prime}, E\right)$-closed absolutely convex hull of any equicontinuous $\sigma\left(E^{\prime}, E\right)$-null sequence in $E^{\prime}$ is $\sigma\left(E^{\prime}, E\right)$-metrisable.

Proof. Any equicontinuous $\sigma\left(E^{\prime}, E\right)$-null sequence in $E^{\prime}$ is the image of the canonical basis $\left(\mathbf{e}_{n}\right)$ in $l^{1}$ under a $\left(\sigma\left(l^{1}, c_{0}\right), \sigma\left(E^{\prime}, E\right)\right)$-continuous linear map. The closed absolutely convex hull of $\left(\mathbf{e}_{n}\right)$ in $l^{1}$ is $\sigma\left(l^{1}, c_{0}\right)$-compact and metrisable, so its image under such a map is $\sigma\left(E^{\prime}, E\right)$-metrisable.

A locally convex space $E$ is separable by seminorm if and only if every continuous linear map from $E$ into a Banach space $F$ has separable range. Let $\mathscr{Z}$ be the operator ideal which consists of the continuous linear maps between Banach spaces that have separable ranges. (For the definition of an operator ideal and related concepts, see [17].) A locally convex topology on a vector space $E$ is an $\mathscr{X}$-topology if it makes $E$ separable by seminorm. We saw above that $\sigma\left(E, E^{\prime}\right)$ is always an $\mathscr{X}$-topology; the associated $\mathscr{X}$-topology on a locally convex space $E$ is defined to be the finest $\mathscr{X}$-topology on $E$ coarser than the given topology. Using Proposition 2.1 we can characterise the associated $\mathscr{X}$-topology as being the topology of uniform convergence on the $\sigma\left(E^{\prime}, E\right)$-metrisable equicontinuous convex subsets of $E^{\prime}$.

Proposition 2.3. Let $\mathscr{T}_{\mathscr{Z}}$ be the associated $\mathscr{R}$-topology on a locally convex space $E$.

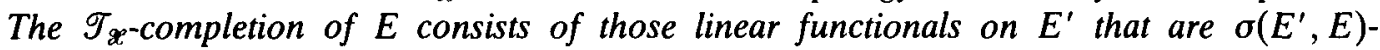
sequentially continuous on the equicontinuous subsets of $E^{\prime}$.

Proof. Using Grothendieck's completeness theorem, we see that the $\mathscr{T}_{\mathscr{x}}$-completion of $E$ consists of the linear functionals on $E^{\prime}$ that are $\sigma\left(E^{\prime}, E\right)$-continuous on the absolutely convex $\sigma\left(E^{\prime}, E\right)$-metrisable equicontinuous subsets of $E^{\prime}$. According to 
Lemma 2.2 , these are precisely the linear functionals on $E^{\prime}$ which are $\sigma\left(E^{\prime}, E\right)$ sequentially continuous on the equicontinuous subsets of $E^{\prime}$.

Thus a locally convex space $E$ is complete in its associated $\mathscr{Z}$-topology if and only if it contains every linear functional that is $\sigma\left(E^{\prime}, E\right)$-sequentially continuous on the equicontinuous subsets of $E^{\prime}$. For any locally convex space $E$, let $d\left(E, E^{\prime}\right)$ be the topology on $E$ of uniform convergence on the $\sigma\left(E^{\prime}, E\right)$-bounded and metrisable subsets of $E^{\prime}$. If the space $E$ is sequentially barrelled (so that all the $\sigma\left(E^{\prime}, E\right)$-null sequences in $E^{\prime}$ are equicontinuous), then $d\left(E, E^{\prime}\right)$ is the associated $\mathscr{X}$-topology on $E$. In [18], T. Kappeler observed that a Banach space $E$ is $d\left(E, E^{\prime}\right)$-complete if and only if it satisfies the condition of Mazur. Proposition 2.3 generalises this observation; by using the associated $\mathscr{X}$-topology instead of $d\left(E, E^{\prime}\right)$, we are able to consider locally convex spaces which are not sequentially barrelled.

At this stage, it is worth mentioning some related work by A. Wilansky [25] on the subject of Mazur spaces. A locally convex space $E$ is a Mazur space if every sequentially continuous linear functional on $E$ is continuous. Obviously a Banach space $E$ satisfies the condition of Mazur if and only if $E^{\prime}$ is a Mazur space when equipped with its weak* topology $\sigma\left(E^{\prime}, E\right)$. In Wilansky's terminology, a Banach space that satisfies the condition of Mazur is a " $\mu B$ space", while a locally convex space $E$ whose dual forms a Mazur space in its $\sigma\left(E^{\prime}, E\right)$-topology is called a " $\mu$ lc space". It is clear that $\mu \mathrm{lc}$ spaces are closely related to the class of locally convex spaces which are complete in their associated $\mathscr{X}$-topologies; the distinction between these two classes of spaces arises from the inclusion of the word "equicontinuous" in the characterisation of the associated $\mathscr{X}$-topology.

Because the associated $\mathscr{X}$-topology $\mathscr{T}_{\mathscr{T}}$ on a locally convex space $E$ is coarser than the given topology, a $\mathscr{T}_{\mathscr{X}}$-complete locally convex space is always complete (cf. [25, 3.1]). It is possible to use Lemma 2.2 to show that if $d\left(E, E^{\prime}\right)$ is coarser than the Mackey topology $\mu\left(E, E^{\prime}\right)$, then $E^{\prime}$ is $\sigma\left(E^{\prime}, E\right)$-sequentially complete (cf. [3, Proposition 3.6]).

Let $E$ and $F$ be Banach spaces, with $F$ satisfying the condition of Mazur. Wilansky has shown $[25,5.1]$ that if there exists a Tauberian operator $T: E \rightarrow F$, then $E$ satisfies the condition of Mazur. We can use our Proposition 1.3 to derive an analogous result for the relation $<$.

Corollary 2.4. Let $E$ and $F$ be locally convex spaces with $E<F$. If $F$ is complete in its associated $\mathscr{X}$-topology, then so is $E$.

From this Corollary we can obtain the desired extension of Edgar's result [8, Proposition 3] concerning Banach spaces satisfying the condition of Mazur.

Proposition 2.5. Let $\mathscr{T}_{\mathscr{X}}$ be the associated $\mathscr{X}$-topology on a locally convex space $E$. Then $E<c_{0}$ if and only if $E$ is $\mathscr{T}_{\mathscr{P}}$-complete.

Proof. Since $c_{0}$ is a separable Banach space, it is complete in its associated $\mathscr{X}$-topology. Therefore if $E<c_{0}$, then $E$ is $\mathscr{T}_{\mathscr{P}}$-complete. To obtain the converse, consider a linear functional $\phi$ on $E^{\prime}$ that does not belong to $E$. If $E$ is $\mathscr{T}_{\mathscr{Z}}$-complete, then $\phi$ must fail to be $\sigma\left(E^{\prime}, E\right)$-sequentially continuous on some equicontinuous convex subset of $E^{\prime}$. In other words, there must be an equicontinuous $\sigma\left(E^{\prime}, E\right)$-null sequence $\left(f_{n}\right)$ in $E^{\prime}$ with $\left(\phi\left(f_{n}\right)\right) \notin c_{0}$. Define $T: E \rightarrow c_{0}$ by $T(x)=\left(f_{n}(x)\right)$; then $\phi \circ T^{\prime}$ fails to be $\sigma\left(l^{1}, c_{0}\right)$ continuous on $l^{1}$, as required. 
A Banach space $E$ is said to be weakly compactly generated (WCG) if it can be expressed as the closed linear span of one of its $\sigma\left(E, E^{\prime}\right)$-compact subsets. We shall show that the class of $\mathscr{T}_{\mathscr{X}}$-complete locally convex spaces contains not only all WCG Banach spaces, but many other spaces besides. Recall that a compact Hausdorff space $K$ is angelic (see [10]) if and only if the closure of each subset $S$ of $K$ is the set of limits of sequences in $S$. Edgar has observed [7, p. 565] that if the closed unit ball of the dual of a Banach space $E$ is $\sigma\left(E^{\prime}, E\right)$-angelic, then $E$ satisfies the condition of Mazur. We can re-formulate this in terms of locally convex spaces as follows.

Proposition 2.6. Let $E$ be a complete locally convex space with associated $\mathscr{X}$-topology $\mathscr{T}_{\mathscr{X}}$. If each $\sigma\left(E^{\prime}, E\right)$-closed equicontinuous subset of $E^{\prime}$ is $\sigma\left(E^{\prime}, E\right)$-angelic, then $E$ is $\mathscr{T}_{\mathscr{x}}$-complete.

Proof. Let $M$ be a $\sigma\left(E^{\prime}, E\right)$-closed equicontinuous subset of $E^{\prime}$. Because $M$ is $\sigma\left(E^{\prime}, E\right)$-angelic, any linear functional on $E^{\prime}$ which is $\sigma\left(E^{\prime}, E\right)$-sequentially continuous on $M$ must be $\sigma\left(E^{\prime}, E\right)$-continuous on $M$. Since $E$ is complete, it follows that every linear functional on $E^{\prime}$ which manages to be $\sigma\left(E^{\prime}, E\right)$-sequentially continuous on the $\sigma\left(E^{\prime}, E\right)$ closed equicontinuous subsets of $E^{\prime}$ is $\sigma\left(E^{\prime}, E\right)$-continuous on $E^{\prime}$. The result now follows by Proposition 2.3 .

Using his "Banach space" version of this Proposition, Edgar was able to deduce that any closed subspace of a WCG Banach space satifises the condition of Mazur. In order to extend this result to locally convex spaces, we turn to some work by R. J. Hunter and J. Lloyd [16]. These authors have made a study of various locally convex analogues of the class of WCG Banach spaces. Amongst other things, they have established the following $[16,2.2(b)]$.

LEMMA 2.7. If a locally convex space $E$ is the closed linear span of the union of a sequence of absolutely convex $\sigma\left(E, E^{\prime}\right)$-compact sets, then every $\sigma\left(E^{\prime}, E\right)$-compact subset of $E^{\prime}$ is $\sigma\left(E^{\prime}, E\right)$-angelic.

Proof. A locally convex space $E$ is of the type mentioned here if and only if there is a metrisable locally convex topology on $E^{\prime}$ coarser than the Mackey topology $\mu\left(E^{\prime}, E\right)$. The result now follows from the "Angelic Lemma" [10, 3.1, 3.10(2)].

This provides us with the following extension of Edgar's result.

Proposition 2.8. If a locally convex space $E$ is the closed linear span of the union of a sequence of absolutely convex $\sigma\left(E, E^{\prime}\right)$-compact sets, then every complete subspace of $E$ is complete in its associated $\mathscr{X}$-topology.

Wilansky also noted that closed subspaces of WCG Banach spaces satisfy the condition of Mazur (see [25, 3.5]). He has defined a compact Hausdorff space $K$ to be a $\mu$-space if $\mathscr{C}(K)$ (the Banach space of all continuous scalar-valued functions on $K$ ) satisfies the condition of Mazur. Recall that a topological space $K$ is Eberlein-compact if it is $\sigma\left(E, E^{\prime}\right)$-homeomorphic to a $\sigma\left(E, E^{\prime}\right)$-compact subset of a Banach space $E$. It is easy to show that a compact Hausdorff space $K$ is Eberlein-compact if and only if $\mathscr{C}(K)$ is a WCG Banach space (see [24, p. 414]). Using this fact, Wilansky was able to deduce that every Eberlein-compact topological space is a $\mu$-space [25, 4.1]. He asked whether the converse is always true. This can be answered in the negative: $M$. Talagrand [24] has 
shown that there exist compact Hausdorff spaces $K$ for which the equicontinuous subsets of $\mathscr{C}(K)^{\prime}$ are $\sigma\left(\mathscr{C}(K)^{\prime}, \mathscr{C}(K)\right)$-angelic, but which fail to be Eberlein-compact. Such a space $K$ is an example of a $\mu$-space that is not Eberlein-compact.

In Wilansky's paper $[25,3.7]$ there is an example (assuming the Continuum Hypothesis) of a Banach space which satisfies the condition of Mazur, but which is not a subspace of any WCG Banach space (see also [5, p. 226]). This provides us with an example of a locally convex space which is complete in its associated $\mathscr{Q}$-topology, but which is not a subspace of the closed linear span of the union of a sequence of absolutely convex weakly compact sets.

In his paper [25], Wilansky also discussed what he called "GB spaces". According to him, these spaces "lie at the opposite end of a spectrum" from the Banach spaces satisfying the condition of Mazur. We shall show that GB spaces are related to compact operators in much the same way as Banach spaces satisfying the condition of Mazur are related to Tauberian operators. However, just as we have chosen to work with

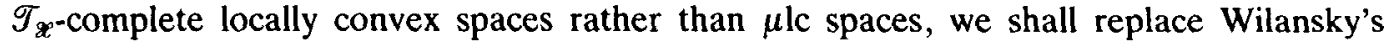
definition of a GB space by one which seems to be better suited to the locally convex situation. In [11], F. J. Freniche has defined a locally convex space $E$ to be a Grothendieck space if the $\sigma\left(E^{\prime}, E\right)$-convergent equicontinuous sequences in $E^{\prime}$ are $\sigma\left(E^{\prime}, E^{\prime \prime}\right)$-convergent. The following result is easy to establish.

Proposition 2.9. For a locally convex space $E$, the following statements are equivalent:

(1) $E$ is a Grothendieck space;

(2) every continuous linear map $T$ from $E$ into a $\mathscr{T}_{\mathscr{X}}$-complete locally convex space $F$ satisfies $T^{\prime \prime}\left(E^{\prime \prime}\right) \subseteq F$;

(3) every continuous linear map $T$ from $E$ into $c_{0}$ satisfies $T^{\prime \prime}\left(E^{\prime \prime}\right) \subseteq c_{0}$.

If a locally convex space $E$ is quasinormable, then a continuous linear map $T$ from $E$ into a Banach space $F$ is weakly compact if and only if $T^{\prime \prime}\left(E^{\prime \prime}\right) \subseteq F[17,17.2 .7]$. We can therefore derive the following version of $[9,3.1]$.

Proposition 2.10. If a locally convex space $E$ is quasinormable, then the following statments are equivalent:

(1) $E$ is a Grothendieck space;

(2) every continuous linear map $T$ from $E$ into a WCG Banach space $F$ is weakly compact;

(3) every continuous linear map $T$ from $E$ into a separable Banach space $F$ is weakly compact;

(4) every continuous linear map $T$ from $E$ into $c_{0}$ is weakly compact.

It is interesting to note the similarity between condition (4) and a result of $M$. Lindström's. A quasinormable locally convex space $E$ is a Schwartz space if and only if every continuous linear map from $E$ into $c_{0}$ is compact [20, p. 426].

3. The Pettis Integral Property. Edgar originally studied Banach spaces satisfying the condition of Mazur because of a connection with Pettis integration. Before we can discuss this topic, it is necessary to introduce a couple of definitions (for further details see [1]). Let $(\Omega, \Sigma, \mu)$ be a probability space, and suppose that $E$ is a locally convex 
space. A map $\Phi: \Omega \rightarrow E$ is bounded if its range $\Phi(\Omega)$ is a bounded subset of $E$. Such a map is weakly $\mu$-measurable if the scalar-valued function $f \circ \Phi$ is $\mu$-measurable for each $f \in E^{\prime}$. We say that a weakly $\mu$-measurable map $\Phi: \Omega \rightarrow E$ is $\mu$-Pettis integrable if for each $S \in \Sigma$, there is an $x \in E$ satisfying

$$
f(x)=\int_{S} f \circ \Phi d \mu \quad \text { for all } f \in E^{\prime} .
$$

A locally convex space $E$ has the $\mu$-Pettis Integral Property if every bounded weakly measurable map $\Phi: \Omega \rightarrow E$ is $\mu$-Pettis integrable. Edgar has shown [8, Proposition 8] that if $E$ and $F$ are Banach spaces with $E<F$, and if $F$ has the $\mu$-Pettis Integral Property for some probability space $(\Omega, \Sigma, \mu)$, then $E$ has the $\mu$-Pettis Integral Property. We can use his proof to obtain a similar result for locally convex spaces.

Proposition 3.1. Let $E$ and $F$ be locally convex spaces with $E<F$. If $F$ has the $\mu$-Pettis Integral Property for some probability space $(\Omega, \Sigma, \mu)$, then so does $E$.

Proof. Let $\Phi: \Omega \rightarrow E$ be a bounded weakly $\mu$-measurable map. For each $S \in \Sigma$, we can define a linear functional $\phi$ on $E^{\prime}$ by

$$
\phi(f)=\int_{S} f \circ \Phi d \mu \text { for all } f \in E^{\prime}
$$

Since $E<F$, we can prove that $\phi \in E$ by showing that $\phi \circ T^{\prime} \in F$ for every continuous linear map $T: E \rightarrow F$. It will then follow that $\Phi$ is $\mu$-Pettis integrable.

Suppose that $T: E \rightarrow F$ is a continuous linear map. Because $T \circ \Phi: \Omega \rightarrow F$ is a bounded weakly $\mu$-measurable map and $F$ has the $\mu$-Pettis Integral Property, $T \circ \Phi$ must be $\mu$-Pettis integrable. Let $y \in F$ be such that

$$
g(y)=\int_{S} g \circ(T \circ \Phi) d \mu \quad \text { for all } g \in F^{\prime} .
$$

Then for each $g \in F^{\prime}$,

$$
\begin{aligned}
\left(\phi \circ T^{\prime}\right)(g) & =\phi\left(T^{\prime}(g)\right) \\
& =\int_{S}\left(T^{\prime}(g)\right) \circ \Phi d \mu \\
& =\int_{S}(g \circ T) \circ \Phi d \mu \\
& =\int_{S} g \circ(T \circ \Phi) d \mu \\
& =g(y) .
\end{aligned}
$$

It follows that $\phi \circ T^{\prime}=y \in F$, as required.

A locally convex space $E$ is said to have the Pettis Integral Property if it has the $\mu$-Pettis Integral Property for every probability space $(\Omega, \Sigma, \mu)$. It can be shown $[1, \mathrm{p}$. 301] that every separable Banach space has the Pettis Integral Property, so in particular $c_{0}$ 
has the Pettis Integral Property. Using Propositions 2.5 and 3.1, we can deduce that whenever a locally convex space $E$ is complete in its associated $\mathscr{Z}$-topology, it has the Pettis Integral Property.

We would like to extend the above result to take account of some recent work by $R$. F. Geitz and F. J. Freniche regarding the average range of a vector measure. Again let $(\Omega, \Sigma, \mu)$ be a probability space. If $E$ is a Banach space, then every bounded weakly $\mu$-measurable function $\Phi: \Omega \rightarrow E$ gives rise to a $\mu$-absolutely continuous vector measure $m_{\Phi}: \Sigma \rightarrow E^{\prime \prime}$. This measure is known as the indefinite Dunford integral of $\Phi$, and is defined for each $S \in \Sigma$ by

$$
f\left(m_{\Phi}(S)\right)=\int_{S} f \circ \Phi d \mu \quad \text { for all } f \in E^{\prime} .
$$

It can be seen that the range of $m_{\Phi}$ is contained in $E$ if and only if $\Phi$ is $\mu$-Pettis integrable. If we now let $E$ be a locally convex space, and consider other vector measures on $(\Omega, \Sigma, \mu)$, then the following question arises. Under what conditions is the range of every $\mu$-absolutely continuous vector measure $v: \Sigma \rightarrow E^{\prime \prime}$ contained in $E$ ?

Let $\overline{c o} A$ denote the $\sigma\left(E^{\prime \prime}, E^{\prime}\right)$-closed convex hull of a subset $A$ of $E^{\prime \prime}$. For each $S \in \Sigma$, put

$$
\Sigma_{S}^{+}=\{A \in \Sigma: A \subseteq S, \mu(A)>0\} .
$$

The average range of a vector measure $v: \Sigma \rightarrow E^{\prime \prime}$ on a measurable set $S \in \Sigma$ is defined to be

$$
\operatorname{AR}_{S}(v)=\left\{\frac{v(A)}{\mu(A)}: A \in \Sigma_{S}^{+}\right\},
$$

In [14], Geitz used this concept to characterise the $\mu$-Pettis integrable functions $\Phi: \Omega \rightarrow E$, where $E$ is a Banach space. It follows from the Hahn-Banach Theorem that in this case

$$
\overline{\mathrm{co}} \mathrm{AR}_{S}\left(m_{\Phi}\right)=\bigcap\{\overline{\mathrm{co}} \varphi(S \backslash B): B \in \Sigma, \mu(B)=0\}
$$

(see [14, 2.2] and [23]). The intersection of this set with $E$ is known as the core of $\Phi$ relative to $S$ (see [1, p. 311]). It follows from Geitz's work that if the map $\Phi: \Omega \rightarrow E$ has non-empty core relative to each $S \in \Sigma_{\Omega}^{+}$, then $\Phi$ is $\mu$-Pettis integrable (see [1, Theorem 7.4.16(d)]).

Following Freniche [12], we shall say that a vector measure $v: \Sigma \rightarrow E^{\prime \prime}$ satisfies Geitz's condition if $E \cap \overline{c o} \operatorname{AR}_{S}(v) \neq \varnothing$ for all $S \in \Sigma_{\Omega}^{+}$. It can be seen that if $E$ is a Banach space, then the indefinite Dunford integral of a bounded weakly measurable function $\Phi: \Omega \rightarrow E$ satisfies Geitz's condition if and only if $\Phi$ has non-empty core relative to each $S \in \Sigma_{\Omega}^{+}$. We shall therefore say that Geitz's condition suffices in $E$ for the probability space $(\Omega, \Sigma, \mu)$ if the range of every $\mu$-absolutely continuous vector measure $v: \Sigma \rightarrow E^{\prime \prime}$ satisfying Geitz's condition is contained in $E$.

Proposition 3.2. Let $E$ and $F$ be locally convex spaces with $E<F$. If Geitz's condition suffices in $F$ for some probability space $(\Omega, \Sigma, \mu)$, then it also suffices in $E$.

Proof. Let $v: \Sigma \rightarrow E^{\prime \prime}$ be a $\mu$-absolutely continuous vector measure. Since $E<F$, we will show that $T^{\prime \prime}(v(S)) \in F$ for each $S \in \Sigma$ and for each continuous linear map $T: E \rightarrow F$. 
First note that for each $T$, the map $v_{T}: \Sigma \rightarrow F^{\prime \prime}$ given by

$$
v_{T}(S)=T^{\prime \prime}(v(S)) \quad \text { for all } S \in \Sigma
$$

is a $\mu$-absolutely continuous vector measure. Furthermore, for each $S \in \Sigma$,

$$
\begin{aligned}
\operatorname{AR}_{S}\left(v_{T}\right) & =\left\{\frac{T^{\prime \prime}(v(A))}{\mu(A)}: A \in \Sigma_{S}^{+}\right\} \\
& =T^{\prime \prime}\left(\operatorname{AR}_{S}(v)\right) .
\end{aligned}
$$

Since $v$ satisfies Geitz's condition,

$$
\begin{aligned}
E \cap \overline{\mathrm{co}} \mathrm{AR}_{S}(v) \neq \varnothing & \Rightarrow F \cap T^{\prime \prime}\left(\overline{\mathrm{co}} \mathrm{AR}_{S}(v)\right) \neq \varnothing \\
& \Rightarrow F \cap \overline{\mathrm{co}} \operatorname{AR}_{S}\left(v_{T}\right) \neq \varnothing
\end{aligned}
$$

for all $S \in \Sigma_{\Omega}^{+}$. Thus $v_{T}$ satisfies Geitz's condition, and so $T^{\prime \prime}(v(S))=v_{T}(S) \in F$ for all $S \in \Sigma$.

Freniche has shown [12, Theorem 1] that if $E$ is a Banach space and $v: \Sigma \rightarrow E^{\prime \prime}$ is a vector measure satisfying Geitz's condition, then for each $S \in \Sigma, v(S)$ is a $\sigma\left(E^{\prime}, E\right)$ sequentially continuous linear functional on $E^{\prime}$. As Freniche points out, this implies that Geitz's condition suffices in every Banach space $E$ satisfying the condition of Mazur. Proposition 3.2 enables us to extend this result to the case when $E$ is a locally convex space that is complete in its associated $\mathscr{X}$-topology. In particular we see that Geitz's condition suffices in $E$ if the $\sigma\left(E^{\prime}, E\right)$-closed equicontinuous subsets of $E^{\prime}$ are $\sigma\left(E^{\prime}, E\right)$-angelic. This last observation generalises another result of Freniche's, which states that Geitz's condition suffices in every Banach space $E$ whose dual unit ball is $\sigma\left(E^{\prime}, E\right)$-sequentially compact.

The following result is a locally convex version of [8, Proposition 6]. For any locally convex space $E$, let $\gamma\left(E, E^{\prime}\right)$ be the topology on $E$ of uniform convergence on the $\sigma\left(E^{\prime}, E\right)$-bounded sequences in $E^{\prime}$. A locally convex space $E$ is $\sigma$-barrelled if and only if $\gamma\left(E, E^{\prime}\right)$ is coarser than the given topology on $E$.

Proposition 3.3. Let $E$ be a $\sigma$-barrelled locally convex space. Then $E<l^{\infty}$ if and only if $E$ is $\gamma\left(E, E^{\prime}\right)$-complete.

Proof. From Proposition 1.3 and the fact that the relation $<$ is functorial, we get that if $E<F$ and $F$ is $\gamma\left(F, F^{\prime}\right)$-complete, then $E$ is $\gamma\left(E, E^{\prime}\right)$-complete. It is easy to see that $l^{\infty}$ is $\gamma\left(l^{\infty},\left(l^{\infty}\right)^{\prime}\right)$-complete, so one half of the equivalence follows.

To see the converse, note that the continuous linear maps from $E$ into $l^{\infty}$ can be identified with the equicontinuous sequences in $E^{\prime}$. If $E$ is $\sigma$-barrelled, then these sequences include all the $\sigma\left(E^{\prime}, E\right)$-bounded sequences in $E^{\prime}$. Suppose that $\phi$ is a linear functional on $E^{\prime}$ that does not belong to $E$. Since $E$ is $\gamma\left(E, E^{\prime}\right)$-complete, $\phi$ must fail to be $\sigma\left(E^{\prime}, E\right)$-continuous on the $\sigma\left(E^{\prime}, E\right)$-closed absolutely convex hull of a $\sigma\left(E^{\prime}, E\right)$ bounded sequence $\left(f_{n}\right)$ in $E^{\prime}$. Let $T: E \rightarrow l^{\infty}$ be defined by $T(x)=\left(f_{n}(x)\right)$ for all $x \in E$. Then $\phi \circ T^{\prime}$ is not $\sigma\left(l^{\infty}, l^{\infty}\right)$-continuous on $l^{\infty \prime}$, as required.

Freniche has shown (see [12, Theorem 3]) that if $E$ is a Banach space, then every $\mu$-absolutely continuous $E^{\prime \prime}$-valued vector measure which satifises Geitz's condition and whose range is not contained in $E$ gives rise to a $\mu$-absolutely continuous $\left(l^{\infty}\right)^{\prime \prime}$-valued 
vector measure which satisfies Geitz's condition and whose range is not contained in $l^{\infty}$. From this fact and Proposition 3.3, we can deduce that if Geitz's condition suffices in $l^{\infty}$ for some probability space $(\Omega, \Sigma, \mu)$, then it suffices in every $\gamma\left(E, E^{\prime}\right)$-complete $\sigma$-barrelled space $E$.

4. Further applications. We shall now explore some further consequences of Proposition 1.3. It should be clear at this stage that even in a Banach space setting, the use of locally convex topologies provides a useful framework for the study of the relation $<$.

G. Godefroy and M. Talagrand [15] have defined a Banach space $E$ to have property $(X)$ if it contains every linear functional $\phi \in E^{\prime \prime}$ which satisfies

$$
\phi\left(\sum_{n=1}^{\infty} f_{n}\right)=\sum_{n=1}^{\infty} \phi\left(f_{n}\right)
$$

for each sequence $\left(f_{n}\right)$ in $E^{\prime}$ that has $\sum_{n=1}^{\infty}\left|f_{n}(x)\right|<\infty$ for all $x \in E$. Edgar [8, Proposition 10] has proved that a Banach space $E$ has property $(\mathrm{X})$ if and only if $E<l^{1}$.

We shall show that property $(X)$ is related to the famous Orlicz-Pettis Theorem (see $[17,14.6 .4]$ and $\left[5\right.$, p. 25]). A sequence $\left(x_{n}\right)$ in a locally convex space $E$ is said to be subseries summable if for each subsequence $\left(x_{n_{k}}\right)$ of $\left(x_{n}\right)$, the series $\sum_{k=1}^{\infty} x_{n_{k}}$ converges in $E$. It can be shown that a sequence $\left(x_{n}\right)$ in $E$ is subseries summable if and only if $\left\{\sum_{n \in N} x_{n}: N\right.$ is a finite subset of $\mathbf{N}\}$ is a relatively $\sigma\left(E, E^{\prime}\right)$-compact subset of $E$ (see $[17,14.6 .4]$ ). It follows that subseries summability is a duality invariant: a sequence is subseries summable with respect to the given topology on $E$ if and only if it is subseries summable with respect to $\sigma\left(E, E^{\prime}\right)$. This last observation is of course a generalisation of the clasic Orlicz-Pettis Theorem, which states that a sequence in a Banach space $E$ is norm-subseries summable if and only if it is $\sigma\left(E, E^{\prime}\right)$-subseries summable. satisfy

For any locally convex space $E$, let $E^{\mathrm{s}}$ consist of those linear functionals $f$ on $E$ that

$$
f\left(\sum_{n=1}^{\infty} x_{n}\right)=\sum_{n=1}^{\infty} f\left(x_{n}\right)
$$

for each subseries summable sequence $\left(x_{n}\right)$ in $E$. Note that $E^{\prime} \subseteq E^{\text {s; }}$; we call $E^{\text {s }}$ the Orlicz-Pettis dual of E.

Let $m_{0}$ be the space of scalar-valued sequences that take on only finitely many values. According to Schur's Lemma (see $[17,10.5 .2])$, the $\sigma\left(l^{1}, m_{0}\right)$-compact sets are exactly the norm-compact subsets of $l^{1}$.

Proposition 4.1. If $\left(x_{n}\right)$ is a sequence in a locally convex space $E$, then the following statements are equivalent:

(1) $\left(x_{n}\right)$ is subseries summable in $E$;

(2) for each $\left(\mu_{n}\right) \in m_{0}$, the series $\sum_{n=1}^{\infty} \mu_{n} x_{n}$ converges in $E$; 
(3) for each $\left(\xi_{n}\right) \in l^{\infty}$, the series $\sum_{n=1}^{\infty} \xi_{n} x_{n}$ is $\mu\left(E, E^{\mathrm{s}}\right)$-convergent;

(4) $\left(x_{n}\right)$ is $\mu\left(E, E^{s}\right)$-subseries summable. $i$, put

Proof. $(1) \Rightarrow(2)$ : Suppose that $\left(\mu_{n}\right) \in m_{0}$ takes on the values $a_{1}, a_{2}, \ldots, a_{m}$. For each

$$
A_{i}=\left\{n \in \mathbf{N}: \mu_{n}=a_{i}\right\}
$$

We then have that $\mathbf{N}=A_{1} \cup A_{2} \cup \ldots \cup A_{m}$. Without any loss of generality, we can assume that each of these sets $A_{i}$ is infinite, so that we can arrange its elements into a strictly increasing sequence $\left(n_{i k}\right)$. Since $\mu_{n_{i k}}=a_{i}$ for all $k \in \mathbf{N}$,

$$
\sum_{k=1}^{\infty} \mu_{n_{i k}} x_{n_{i k}}=a_{i} \sum_{k=1}^{\infty} x_{n_{i k}} .
$$

(The series on the right converges in $E$ because of the subseries summability of $\left(x_{n}\right)$.) By considering partial sums, we see that the series $\sum_{n=1}^{\infty} \mu_{n} x_{n}$ converges in $E$, with

$$
\sum_{n=1}^{\infty} \mu_{n} x_{n}=\sum_{i=1}^{m} \sum_{k=1}^{\infty} a_{i} x_{n_{i k}} \text {. }
$$

(2) $\Rightarrow(3)$ : First note that if $\sum_{n=1}^{\infty} \mu_{n} x_{n}$ converges in $E$ for every $\left(\mu_{n}\right) \in m_{0}$, then for each such $\left(\mu_{n}\right)$ the sequence $\left(\mu_{n} x_{n}\right)$ is subseries summable in $E$. By the definition of $E^{\mathrm{s}}$, it follows that

$$
f\left(\sum_{n=1}^{\infty} \mu_{n} x_{n}\right)=\sum_{n=1}^{\infty} \mu_{n} f\left(x_{n}\right) \quad \text { for all } f \in E^{\mathrm{s}}
$$

Thus the series $\sum_{n=1}^{\infty} \mu_{n} x_{n}$ is $\sigma\left(E, E^{\mathrm{s}}\right)$-convergent. By considering sequences $\left(\mu_{n}\right)$ that take on the values 0 and 1 , we see that $\left(x_{n}\right)$ is $\sigma\left(E, E^{\mathrm{s}}\right)$-subseries summable in $E$. Thus for each $f \in E^{\mathrm{s}}, \sum_{n=1}^{\infty} f\left(x_{n}\right)$ is an unconditionally convergent series of scalars. Such a series is always absolutely convergent, which means that $\left(f\left(x_{n}\right)\right) \in l^{1}$. We can therefore define a $\operatorname{map} T: E^{\mathrm{s}} \rightarrow l^{1}$ by

$$
T(f)=\left(f\left(x_{n}\right)\right) \quad \text { for all } f \in E^{\mathrm{s}} .
$$

This map has an adjoint $T^{\prime}: m_{0} \rightarrow E$ given by

$$
T^{\prime}\left(\left(\mu_{n}\right)\right)=\sum_{n=1}^{\infty} \mu_{n} x_{n} \quad \text { for all }\left(\mu_{n}\right) \in m_{0} .
$$

It follows that $T: E^{\mathrm{s}} \rightarrow l^{1}$ is $\left(\sigma\left(E^{\mathrm{s}}, E\right), \sigma\left(l^{1}, m_{0}\right)\right)$-continuous, and therefore maps $\sigma\left(E^{\mathrm{s}}, E\right)$-compact subsets of $E^{\mathrm{s}}$ onto $\sigma\left(l^{1}, m_{0}\right)$-compact subsets of $l^{1}$. According to Schur's Theorem, the $\sigma\left(l^{1}, m_{0}\right)$-compact subsets of $l^{1}$ are norm-compact, so this means that $T$ maps the absolutely convex $\mu\left(E, E^{\mathrm{s}}\right)$-equicontinuous subsets of $E^{\mathrm{s}}$ onto relatively norm-compact subsets of $l^{1}$. Taking adjoints, we find that $T^{\prime}$ maps the absolutely convex 
$\mu\left(l^{1}, l^{\infty}\right)$-equicontinuous subsets of $m_{0}$ onto relatively $\mu\left(E, E^{\mathrm{s}}\right)$-compact subsets of $E$. The sections of each sequence $\left(\xi_{n}\right) \in l^{\infty}$ form a $\mu\left(l^{1}, l^{\infty}\right)$-equicontinuous subset of $m_{0}$; the image of this set under $T^{\prime}$ is the set of all partial sums of $\sum_{n=1}^{\infty} \xi_{n} x_{n}$. Since we have shown that the latter set is relatively $\mu\left(E, E^{\mathrm{s}}\right)$-compact in $E$, the series $\sum_{n=1}^{\infty} \xi_{n} x_{n}$ is $\mu\left(E, E^{\mathrm{s}}\right)$ convergent.

(3) $\Rightarrow(4)$ : Given a subsequence $\left(x_{n_{k}}\right)$ of $\left(x_{n}\right)$, define $\left(\xi_{m}\right) \in l^{\infty}$ by

$$
\xi_{m}= \begin{cases}1 & \text { if } m=n_{k} \text { for some } k \in \mathbf{N} \\ 0 & \text { otherwise. }\end{cases}
$$

Clearly $\sum_{k=1}^{\infty} x_{n_{k}}=\sum_{n=1}^{\infty} \xi_{n} x_{n}$; the latter series $\mu\left(E, E^{\mathrm{s}}\right)$-converges in $E$.

(4) $\Rightarrow(1)$ : This follows immediately from the fact that $\mu\left(E, E^{\mathrm{s}}\right)$ is finer than the given topology on $E$.

The topology on a locally convex space $E$ is defined to be an Orlicz-Pettis topology if there is no strictly finer locally convex topology on $E$ with the same subseries summable sequences (see $[4,4.1]$ ). Using the above Proposition, we can see that the topology $\mathscr{T}$ on $E$ is an Orlicz-Pettis topology if and only if it equals $\mu\left(E, E^{s}\right)$. In other words, $\mathscr{T}$ is an Orlicz-Pettis topology if and only if it is the Mackey topology on $E$ and every linear functional in $E^{\mathrm{s}}$ belongs to $E^{\prime}$.

Proposition 4.2. Let $E$ and $F$ be locally convex spaces, with $E<F$. If $\mu\left(F^{\prime}, F\right)$ is an Orlicz-Pettis topology, then so is $\mu\left(E^{\prime}, E\right)$.

Proof. We have to show that when $E^{\prime}$ is equipped with the topology $\sigma\left(E^{\prime}, E\right)$, its Orlicz-Pettis dual is $E$. Suppose that $\phi$ is a linear functional on $E^{\prime}$ which satisfies

$$
\phi\left(\sum_{n=1}^{\infty} f_{n}\right)=\sum_{n=1}^{\infty} \phi\left(f_{n}\right)
$$

for each $\sigma\left(E^{\prime}, E\right)$-subseries summable sequence $\left(f_{n}\right)$ in $E^{\prime}$. Since $E<F$, we can show that $\phi \in E$ by proving that $\phi \circ T^{\prime} \in F$ for each continuous linear map $T: E \rightarrow F$. Let $\left(g_{n}\right)$ be a $\sigma\left(F^{\prime}, F\right)$-subseries summable sequence in $F^{\prime}$. For each continuous linear map $T: E \rightarrow F$, the sequence $\left(T^{\prime}\left(g_{n}\right)\right)$ is $\sigma\left(E^{\prime}, E\right)$-subseries summable, so that

$$
\begin{aligned}
\phi \circ T^{\prime}\left(\sum_{n=1}^{\infty} g_{n}\right) & =\phi\left(\sum_{n=1}^{\infty} T^{\prime}\left(g_{n}\right)\right) \\
& =\sum_{n=1}^{\infty} \phi \circ T^{\prime}\left(g_{n}\right) .
\end{aligned}
$$

Since $\mu\left(F^{\prime}, F\right)$ is an Orlicz-Pettis topology, it follows that $\phi \circ T^{\prime} \in F$, as required.

We can now generalise Edgar's result [8, Proposition 10] regarding Banach spaces with property $(\mathrm{X})$.

Proposition 4.3. Let $E$ be a locally convex space with the property that every $\sigma\left(E^{\prime}, E\right)$-subseries summable sequence in $E^{\prime}$ is equicontinuous on $E$. Then $\mu\left(E^{\prime}, E\right)$ is an Orlicz-Pettis topology if and only if $E<l^{1}$. 
Proof. One half of this equivalence follows from the fact that $\mu\left(l^{\infty}, l^{1}\right)$ is an Orlicz-Pettis topology; this in turn follows from the proof of Proposition 4.1. The converse hinges on the fact that for any locally convex space $E$, the continuous linear maps $T: E \rightarrow l^{1}$ are in one-to-one correspondence with the equicontinuous $\sigma\left(E^{\prime}, E\right)$ subseries summable sequences in $E^{\prime}$.

COROLlary 4.4. Let $E$ be a locally convex space with the property that every $\sigma\left(E^{\prime}, E\right)$-subseries summable sequence in $E^{\prime}$ is equicontinuous on $E$. If $\mu\left(E^{\prime}, E\right)$ is an Orlicz-Pettis topology, then $E$ is complete in its associated $\mathscr{X}$-topology.

The following example illustrates why it is necessary to specify that the $\sigma\left(E^{\prime}, E\right)$ subseries summable sequences in $E^{\prime}$ be equicontinuous on $E$. Consider an infinitedimensional Banach space $E$ that has property (X). According to the above Proposition, $\mu\left(E^{\prime}, E\right)$ is an Orlicz-Pettis topology. However when we equip the same space $E$ with its weak topology $\sigma\left(E, E^{\prime}\right)$, we obtain a locally convex space for which $\mu\left(E^{\prime}, E\right)$ is an Orlicz-Pettis topology, but which is not itself complete in its associated $\mathscr{X}$-topology.

The relation $<$ can be used to formulate a variety of similar results involving the Mackey topology $\mu\left(E^{\prime}, E\right)$. Let $\langle E, F\rangle$ be a dual pair of vector spaces. A sequence $\left(x_{n}\right)$ in $E$ is said to be locally-null if it converges to 0 in the normed space generated by a $\sigma(E, F)$-bounded absolutely convex subset $B$ of $E$. Let $v(F, E)$ denote the topology on $F$ of uniform convergence on the locally-null sequences in $E$. It can be shown $[17,13.2 .4]$ that the topology on a locally convex space $E$ is bornological if and only if it is the Mackey topology $\mu\left(E, E^{\prime}\right)$ on $E$ and $E^{\prime}$ is $v\left(E^{\prime}, E\right)$-complete. We will use the "dual" version of this result: $\mu\left(E^{\prime}, E\right)$ is a bornological topology if and only if $E$ is $v\left(E, E^{\prime}\right)$-complete.

Propostrion 4.5. Let $E$ and $F$ be locally convex spaces with $E<F$. If $\mu\left(F^{\prime}, F\right)$ is a bornological topology, then so is $\mu\left(E^{\prime}, E\right)$.

Proof. Since the image of a locally-null sequence under a continuous linear map is again locally-null, every continuous linear map $T: E \rightarrow F$ is $\left(v\left(E, E^{\prime}\right), v\left(F, F^{\prime}\right)\right)$ continuous. The result thus follows by Proposition 1.3 and the fact that the relation $<$ is functorial.

If the bounded set $B$ used in the above definition of a locally-null sequence is a Banach disc (so that the normed space generated by $B$ is complete), then the sequence $\left(x_{n}\right)$ is said to be fast-null. Just as before, the topology on a locally convex space $E$ is ultrabornological if and only if it equals the Mackey topology $\mu\left(E, E^{\prime}\right)$ on $E$ and $E^{\prime}$ is complete with respect to the topology of uniform convergence on the fast-null sequences in $E$.

Proposition 4.6. Let $E$ and $F$ be locally convex spaces with $E<F$. If $\mu\left(F^{\prime}, F\right)$ is an ultrabornological topology, then so is $\mu\left(E^{\prime}, E\right)$.

It is easy to modify the proof of Proposition 1.3 to show that if $E<F$ and $F$ is sequentially complete, then $E$ is sequentially complete. Again appealing to the fact that $<$ is functorial, we obtain the following extension of [8, Proposition 9].

Proposition 4.7. Let $E$ and $F$ be locally convex spaces with $E<F$. If $F$ is $\sigma\left(F, F^{\prime}\right)$-sequentially complete, then $E$ is $\sigma\left(E, E^{\prime}\right)$-sequentially complete. 


\section{REFERENCES}

1. R. D. Bourgin, Geometric aspects of convex sets with the Radon-Nikodým property, Lecture Notes in Mathematics 993 (Springer-Verlag, 1983).

2. H. Buchwalter, Espaces localement convex semi-faibles, lleme Coll. Anal. Fonct. Bordeaux 1973, Pub. Dep. Math. Lyon 10 (1973).

3. P. Dierolf, Theorems of the Orlicz-Pettis type for locally convex spaces, Manuscripta Math. 20 (1977), 73-94.

4. P. Dierolf and S. Dierolf, Topological properties of the dual pair $\left\langle\mathscr{B}(\Omega)^{\prime}, \mathscr{B}(\Omega)^{\prime \prime}\right\rangle$, Pacific J. Math. 108 (1983), 51-82.

5. J. Diestel, Sequences and series in Banach spaces, Graduate Texts in Mathematics 92 (Springer-Verlag, 1984).

6. L. Drewnowski, Another note on Kalton's theorem, Studia Math. 52 (1975), 233-237. $559-579$.

7. G. A. Edgar, Measurability in a Banach space II, Indiana Univ. Math. J. 28 (1979),

8. G. A. Edgar, An ordering for the Banach spaces, Pacific J. Math. 108 (1983), 83-98.

9. B. T. Faires, Varieties and vector measures, Math. Nachr. 85 (1978), 303-314. $1980)$.

10. K. Floret, Weakly compact sets, Lecture Notes in Mathematics 801 (Springer-Verlag,

11. F. J. Freniche, Grothendieck locally convex spaces of continuous vector valued functions, Pacific J. Math. 120 (1985), 345-355.

12. F. J. Freniche, Some remarks on the average range of a vector measure, Proc. Amer. Math. Soc. 107 (1989), 119-124.

13. H. G. Garnir, M. De Wilde and J. Schmets, Analyse Fonctionelle, Tome I, (BirkhäuserVerlag, 1968).

14. R. F. Geitz, Geometry and the Pettis integral, Trans. Amer. Math. Soc. (2) 269 (1982), $535-548$.

15. G. Godefroy and M. Talagrand, Classes d'espaces de Banach à prédual unique. C.R. Acad. Sci. Paris 292 (1981), 323-325.

16. R. J. Hunter and J. Lloyd, Weakly compactly generated locally convex spaces, Math. Proc. Cambr. Philos. Soc. 82 (1977), 85-98.

17. H. Jarchow, Locally convex spaces, (Teubner, 1981).

18. T. Kappeler, Banach spaces with the condition of Mazur, Math. Z. 191 (1986), 623-631.

19. N. Kalton and A. Wilansky, Tauberian operators on Banach spaces, Proc. Amer. Math. Soc. 57 (1976), 251-255.

20. M. Lindström, A characterization of Schwartz spaces, Math. Z. 198 (1988), 423-430.

21. R. D. Neidinger, Concepts in the real interpolation of Banach spaces, Functional Analysis: Proceedings of the Seminar at the University of Texas at Austin, 1986-87 (E. Odell and H. Rosenthal, eds), 43-53, Lecture Notes in Mathematics 1332 (Springer-Verlag, 1988).

22. H. Pfister, Bemerkungen zum Satz über die Separabilität der Fréchet-Montel-Räume, Arch. Math. (Basel) 27 (1976), 86-92.

23. F. D. Sentilles and R. F. Wheeler, Pettis integration via the Stonian transform, Pacific J. Math. 107 (1983), 473-496. 407-438.

24. M. Talagrand, Espaces de Banach faiblement $\mathscr{K}$-analytiques, Ann. Math. 110 (1979),

25. A. Wilansky, Mazur spaces, Intern. J. Math. Math. Sci. 4 (1981), 39-55.

\section{Department of Mathematics}

UNIVERSITY OF CAPE TOWN

RONDEBOSCH

7700 South Africa 